Stud. Univ. Babeş-Bolyai Math. 66(2021), No. 2, 247-255

DOI: 10.24193/subbmath.2021.2.01

\title{
Graph-directed random fractal interpolation function
}

\author{
Ildikó Somogyi and Anna Soós
}

Dedicated to Professor Gheorghe Coman on the occasion of his 85th anniversary.

\begin{abstract}
Barnsley introduced in [1] the notion of fractal interpolation function (FIF). He said that a fractal function is a (FIF) if it possess some interpolation properties. It has the advantage that it can be also combined with the classical methods or real data interpolation. Hutchinson and Rüschendorf [7] gave the stochastic version of fractal interpolation function. In order to obtain fractal interpolation functions with more flexibility, Wang and Yu [9] used instead of a constant scaling parameter a variable vertical scaling factor. Also the notion of fractal interpolation can be generalized to the graph-directed case introduced by Deniz and Özdemir in [5]. In this paper we study the case of a stochastic fractal interpolation function with graph-directed fractal function.
\end{abstract}

Mathematics Subject Classification (2010): 28A80, 60G18.

Keywords: Fractal interpolation function, iterated function system, random fractal interpolation function.

\section{Introduction}

In the construction of a fractal interpolation function Barnsley used the theory of iterated function system [1], [3],[2]. For this we will consider two separable metric spaces $\left(X, d_{X}\right)$ and $\left(Y, d_{Y}\right)$ and a given collection of $N$ bijections $L_{i}: X \rightarrow X_{i}$ such that

$$
\begin{gathered}
\left\{X_{i}=L_{i}(X) \mid i \in\{1,2, . . N\}\right\} \\
\cup_{i=1}^{N} X_{i}=X \quad \text { and } \quad \operatorname{int}\left(X_{i}\right) \cap \operatorname{int}\left(X_{j}\right)=\emptyset, \quad \text { for } \quad i \neq j .
\end{gathered}
$$

For $g_{i}: X_{i} \rightarrow Y, i \in\{1,2, . . N\}$, define $\sqcup_{i} g_{i}: X \rightarrow Y$ by

$$
\left(\sqcup_{i} g_{i}\right)(x)=g_{j}(x) \quad \text { for } \quad x \in X_{j} .
$$

Assume that mappings $F_{i}: X \times Y \rightarrow Y, F_{i}(x, \cdot) \in \operatorname{Lip}^{<1}(Y), x \in X$ are given, $i \in\{1,2, \ldots N\}$, where $\operatorname{Lip}^{<1}(Y)$ is the set of all Lipschitz functions with Lipschitz 
constant less that 1 .

Let $\mathbf{F}=\left\{F_{1}, F_{2}, \ldots, F_{N}\right\}$, then $\{X, \mathbf{F}\}$ is a so-called Iterated Function System (IFS). Denote $\alpha_{i}=L i p F_{i}$.

For $f: X \rightarrow Y$, define the operator $\mathbf{F}: L_{\infty}(X, Y) \rightarrow Y^{X}$ by

$$
\mathbf{F} f=\sqcup_{i} F_{i}\left(L_{i}^{-1}, f \circ L_{i}^{-1}\right) .
$$

Then $f$ is a selfsimilar fractal function if $\mathbf{F} f=f$.

Let $\Gamma:=\left\{\left(x_{0}, y_{0}\right), \ldots,\left(x_{N}, y_{N}\right) \in(X \times Y)\right\}$ be the set of interpolation points.

A fractal function $f$ has the interpolation properties with respect to $\Gamma$ if

$$
f\left(x_{j}\right)=y_{j} \quad \text { for all } \quad j=0,1, \ldots, N .
$$

Denote

$$
C^{*}(X, Y):=\left\{f \in C(X, Y) \mid \quad f\left(x_{j}\right)=y_{j}, \quad j \in\{1,2, \ldots, N\}\right\} .
$$

Theorem 1.1 (Barnsley, [2]). Let $\Gamma$ be a set of interpolation points and let $\{X, \mathbf{F}\}$ be the IFS. Suppose

$$
F_{i}\left(x_{0}, y_{0}\right)=y_{i-1}, \quad F_{i}\left(x_{N}, y_{N}\right)=y_{i}
$$

for all $i \in\{1,2, \ldots, N\}$ and $\alpha_{\infty}:=\max \alpha_{i}<1$. Then there exists a selfsimilar fractal function $f^{*} \in C^{*}(X, Y)$ such that $\mathbf{F} f^{*}=f^{*}$.

In order to obtain more various (FIF) in many papers the classical interpolation methods are combined with these fractal interpolation functions, [4],[8].

\section{Stochastic fractal interpolation function}

Let $(\Omega, \mathcal{K}, P)$ be a probability space and $\Gamma:=\left\{\left(x_{i}, y_{i}\right), i=0,1, \ldots, N\right\}$ be a set of interpolation points in $X \times Y$.

Let $L_{i}: X \rightarrow X$ be contractiv Lipschitz maps such that $L_{i}\left(x_{0}\right)=x_{i-1}$ and $L_{i-1}\left(x_{N}\right)=x_{i}$ for all $i \in\{1, \ldots, N\}$.

The IFS $\{X, \mathbb{F}\}$ is defined by $F_{i}: X \times Y \rightarrow Y$ such that $F_{i}(x, \cdot) \in \operatorname{Lip}^{<1}(Y)$ for all $x \in X$ and

and

$$
F_{i}\left(x_{0}, y_{0}\right)=y_{i-1} \quad \text { with probability } 1 \text { (a.s.) }
$$

$$
F_{i}\left(x_{N}, y_{N}\right)=y_{i} \quad \text { with probability } 1 \text { (a.s.) }
$$

for all $i \in\{1, \ldots, N\}$.

$$
F_{i}(x, y)=\alpha_{i} y+q_{i}(x), i=1,2, . ., N,
$$

where $\alpha_{i}$ are random variables defined on $\Omega$ satisfying

$$
\left\|\alpha_{i}\right\|_{\infty}=\sup \left\{\left|\alpha_{i}(\omega)\right|: \omega \in \Omega\right\}<1, \quad i=1,2, \ldots, N .
$$

The random function $\mathbb{F}$ is defined up to probability distribution by

$$
\mathbb{F} f=\sqcup_{i} F_{i}\left(L_{i}^{-1}, f^{(i)} \circ L_{i}^{-1}\right),
$$

where $\mathbb{F}, f^{(1)}, \ldots, f^{(N)}$ are independent and $f^{(i)} \stackrel{d}{=} f$, for $i=1,2, \ldots, N$.

We say $f$ is a random fractal function, if

$$
\mathbb{F} f \stackrel{d}{=} f
$$


and it has the interpolation properties with respect to $\Gamma$ if $f\left(x_{i}\right)=y_{i}$ a. s. for all $i \in\{0,1, \ldots, N\}$.

We will consider

$$
C_{\omega}(X, Y):=\{f: \Omega \times X \rightarrow Y, f \text { continuous a.s. }\}
$$

and

$$
\begin{gathered}
C_{\omega}^{*}(X, Y):=\left\{g \in C_{\omega}(X, Y) \mid g\left(x_{i}\right)=y_{i} \text { a.s. }, i \in\{1, \ldots, N\}\right\} . \\
\mathbb{L}_{\infty}:=\left\{g: \Omega \times X \rightarrow Y \mid \text { ess } \sup _{\omega} \text { ess } \sup _{x} d_{Y}\left(g^{\omega}(x), a\right)<\infty\right\}
\end{gathered}
$$

for some $a \in X$.

For $f, g \in \mathbb{L}_{\infty}$ we define

$$
d_{\infty}^{*}(f, g):=e s s \sup _{\omega} d_{\infty}\left(f^{\omega}, g^{\omega}\right)
$$

where

$$
d_{\infty}(f, g)=e s s \sup _{x} d_{Y}(f(x), g(x)) .
$$

Theorem 2.1. Let $\Gamma$ be a set of interpolation points in $X \times Y$ and let $\{X, \mathbb{F}\}$ be the IFS defined above. If $\lambda_{\infty}:=$ ess $\sup _{\omega} \max _{i} \alpha_{i}^{\omega}<1$ and

$$
\text { ess } \sup _{\omega} \max _{i} d_{Y}\left(F_{i}(a, f(a)), a\right)<\infty
$$

for some $a \in X$, then there exists $f^{*} \in C_{\omega}^{*}(X, Y)$ such that $\mathbb{F} f^{*}=f^{*}$. Moreover, $f^{*}$ is unique up to probability distribution.

Example 2.2. $X=[0,1], Y=\mathbb{R}, N>0$.

$$
\begin{gathered}
\Gamma:=\left\{\left(x_{i}, y_{i}\right) \in[0,1] \times \mathbb{R} \mid 0=x_{0}<x_{1}<\ldots<x_{N}=1\right\} . \\
L_{i}: X \rightarrow X_{i}, \quad L_{i}(x):=a_{i} x+d_{i}, a_{i}, d_{i} \in \mathbb{R}, \quad i \in\{1,2, \ldots, N\} . \\
F_{i}: X \times Y \rightarrow Y, i=\{1,2, . ., N\}, \\
F_{i}(x, y):=\alpha_{i} y+q_{i}(x), q_{i}(x)=c_{i} x+e_{i},
\end{gathered}
$$

$\alpha_{i}$ is a random variable, $\lambda_{\infty}:=$ ess $\sup _{\omega} \max _{i} \alpha_{i}<1$.

We can compute $a_{i}, c_{i}, d_{i}, e_{i}$ by the conditions $L_{i}\left(x_{0}\right)=x_{i-1}, \quad L_{i}\left(x_{N}\right)=x_{i}$

$$
F_{i}\left(x_{0}, y_{0}\right)=y_{i-1}, \quad F_{i}\left(x_{N}, y_{N}\right)=y_{i} \quad \text { a.s. }
$$

for all $i \in\{1, \ldots, N\}$.

$W_{i}: X \times Y \rightarrow X \times Y W_{i}(x, y)=\left(L_{i}(x), F_{i}(x, y)\right), i \in\{1,2,, \ldots, N\}$.

Using $\mathbb{W}:=\left(W_{1}, \ldots, W_{N}\right), \operatorname{IFS}\{X, \mathbb{W}\}$

$$
\mathbb{W}_{i}: X \times Y \rightarrow L \times Y, \quad \mathbb{W}_{i}(x, y)=\left(L_{i}(x), F_{i}(x, y)\right) \quad i=1,, \ldots, N,
$$

for any $K_{0} \subset X \times U$

$$
K_{n}=\mathbb{W} K_{n-1}=\cup_{i=0}^{N} W_{i}^{\omega} K_{n-1}=\mathbb{W}^{n}\left(K_{0}\right) .
$$

Then

$$
\text { ess } \sup _{\omega} d_{H}\left(\mathbb{W}^{n}\left(K_{0}\right), \text { graph } f^{*}\right) \rightarrow 1
$$

as $n \rightarrow \infty, d_{H}$ denotes the Hausdorff distance. 


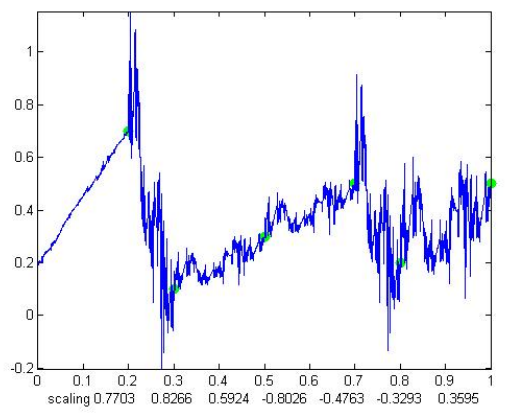

FiguRE 1. Fractal interpolation function with variable parameter, $\{(0,0.2),(0.2,0.7),(0.3,0.1),(0.5,0.3),(0.7,0.5),(0.8,0.2),(1,0.5)\}$

\section{Graph directed fractal interpolation function}

Let $\mathcal{G}=(V, E)$ be a graph, $V$ is the set of vertices and $E$ is the set of edges. For $\alpha, \beta \in V$, let $E^{\alpha, \beta}$ be the set of edges from $\alpha$ to $\beta$, and $K^{\alpha, \beta}$ is the number of elements of $E^{\alpha, \beta}$. Also let $\left\{X^{\alpha} \mid \alpha \in V\right\}$ be a set of complete metric spaces and $\phi_{i}^{\alpha \beta}: X^{\beta} \rightarrow X^{\beta}$ are contraction mappings, for $i=1,2, \ldots, K^{\alpha \beta}$. Then from [6] it follows that there exists a unique family of nonempty compact sets $A^{\alpha} \subset X^{\alpha}$ such that $A^{\alpha}=\cup_{\beta \in V} \cup_{i=1}^{k} \phi_{i}^{\alpha \beta}\left(A^{\beta}\right)$. Then $\left\{X^{\alpha}, \phi_{i}^{\alpha \beta}\right\}$ is a graph-directed iterated function system. Let

$$
\Gamma^{p}=\left\{\left(x_{0}^{p}, y_{0}^{p}\right),\left(x_{1}^{p}, y_{1}^{p}\right), \ldots,\left(x_{N_{p}}^{p}, y_{N_{p}}^{p}\right)\right\}
$$

be the data sets in $\mathbb{R}^{2}$, where $N_{p} \geq 2$, for all $p=1,2, \ldots, n$. These data points satisfy the following condition in order that the maps from the iterated function system to be contractions:

$$
\frac{x_{i}^{l}-x_{i-1}^{l}}{x_{N_{p}}^{p}-x_{0}^{p}}<1,
$$

for all $p \neq l, \quad p, l=1,2, \ldots, n, \quad i=1,2, \ldots, N_{l}$. In [5] we can find the proof regarding the existence of a graph-directed fractal function:

Theorem 3.1. If we condsider the data set $\Gamma^{p}$ in $\mathbb{R}^{2}$ for $p=1,2, \ldots, n$ satisfying (3.2), then there exists a graph-directed iterated function system, with attractors $A_{p}, p=$ $1,2, \ldots, n$, such that $A_{p}$ is the graph of a function which interpolates the data set $\Gamma^{p}$ for each $p$.

In the case $n=2$ the construction of these iterated function systems can be done using the method given in [5].

\section{Graph directed random fractal interpolation function}

Let $(\Omega, \mathcal{K}, P)$ be a probability space and $\left\{X^{\alpha} \mid \alpha \in V\right\}$ a set of complete separable metric spaces and $\Phi_{i}^{\alpha \beta}: \Omega \times X^{\beta} \rightarrow X^{\alpha}$ are random variables. Then there exists 
$A^{\alpha} \subseteq \Omega \times X^{\alpha}$ defined up to probability distribution by

$$
A^{\alpha} \stackrel{d}{=} \cup_{\beta \in V} \cup_{i=1}^{k} \Phi_{i}^{\alpha \beta}\left(A^{\beta}\right) .
$$

The system $\left\{\Omega \times X^{\alpha}, \Phi_{i}^{\alpha \beta}\right\}$ is the graph directed random iterated function system and $A^{\alpha}$ is the attractor of the system.

Theorem 4.1. Let $\Gamma^{p}=\left\{\left(x_{0}^{p}, y_{0}^{p}\right),\left(x_{1}^{p}, y_{1}^{p}\right), \ldots,\left(x_{N_{p}}^{p}, y_{N_{p}}^{p}\right)\right\}$ be the data sets in $\mathbb{R}^{2}$ which satisfies (3.2), then there exists a graph directed random iterated function system with attractor $A^{\alpha}$ such that $A^{\alpha}$ is the graph of a random function which interpolates $\Gamma^{\alpha}$ for each $\alpha$.

Proof. We will construct a graph directed random iterated function system for which Theorem 2 holds. Let $n=2$ and

where $N, M \geq 2$. Suppose

$$
\begin{aligned}
& \Gamma^{1}=\left\{\left(x_{0}^{1}, y_{0}^{1}\right), \ldots,\left(x_{N}^{1}, y_{N}^{1}\right)\right\}, \\
& \Gamma^{2}=\left\{\left(x_{0}^{2}, y_{0}^{2}\right), \ldots,\left(x_{M}^{2}, y_{M}^{2}\right)\right\},
\end{aligned}
$$

$$
\frac{x_{i}^{1}-x_{i-1}^{1}}{x_{M}^{2}-x_{0}^{2}}<1 \text { and } \frac{x_{j}^{2}-x_{j-1}^{2}}{x_{N}^{1}-x_{0}^{1}}<1
$$

$\forall i=1, \ldots, N, \quad j=1, \ldots, M$.

Let $\mathcal{G}=(V, E)$ such that $V=\{1,2\}$ and $K^{11}+K^{12}=N, K^{21}+K^{22}=M$ and $\Phi_{i}^{\alpha \beta}: \Omega \times \mathbb{R}^{2} \rightarrow \mathbb{R}^{2}, i=1, \ldots, K^{\alpha \beta}, \alpha, \beta \in\{1,2\}$

$$
\Phi_{i}^{\alpha \beta}(x, y)=\left(\begin{array}{cc}
a_{i}^{\alpha \beta} & 0 \\
c_{i}^{\alpha \beta} & d_{i}^{\alpha \beta}
\end{array}\right)\left(\begin{array}{l}
x \\
y
\end{array}\right)+\left(\begin{array}{c}
e_{i}^{\alpha \beta} \\
f_{i}^{\alpha \beta}
\end{array}\right) .
$$

Suppose

$$
\begin{gathered}
\left\{\begin{aligned}
\Phi_{i}^{11}\left(x_{0}^{1} y_{0}^{1}\right)= & \left(x_{i-1}^{1}, y_{i-1}^{1}\right) \text { a.s. } \\
\Phi_{i}^{11}\left(x_{N}^{1} y_{N}^{1}\right)= & \left(x_{i}^{1}, y_{i}^{1}\right) \text { for } i=1,2, \ldots, K^{11}
\end{aligned}\right. \\
\left\{\begin{aligned}
\Phi_{i-k^{11}}^{12}\left(x_{0}^{2} y_{0}^{2}\right)= & \left(x_{i-1}^{1}, y_{i-1}^{1}\right) \text { a.s. } \\
\Phi_{i-K^{11}}^{12}\left(x_{M}^{2} y_{M}^{2}\right)= & \left(x_{i}^{1}, y_{i}^{1}\right) \text { for } i=K^{11}+1, \ldots, N
\end{aligned}\right. \\
\left\{\begin{array}{cc}
\Phi_{i}^{21}\left(x_{0}^{1} y_{0}^{1}\right)= & \left(x_{i-1}^{2}, y_{i-1}^{2}\right) \text { a.s. } \\
\Phi_{i}^{21}\left(x_{N}^{1} y_{N}^{1}\right)= & \left(x_{i}^{2}, y_{i}^{2}\right) \text { for } i=1,2, \ldots, K^{21}
\end{array}\right. \\
\begin{cases}\Phi_{i-K^{21}}^{22}\left(x_{0}^{2} y_{0}^{2}\right)= & \left(x_{i-1}^{2}, y_{i-1}^{2}\right) \text { a.s. } \\
\Phi_{i-K^{21}}^{22}\left(x_{M}^{2} y_{M}^{2}\right)= & \left(x_{i}^{2}, y_{i}^{2}\right) \text { for } i=K^{21}, \ldots, M .\end{cases}
\end{gathered}
$$

$\forall i=1, \ldots, K^{11}$.

From these conditions we have the following equations:

$$
\begin{cases}x_{i-1}^{1}= & a_{i}^{11} x_{0}^{1}+e_{i}^{11} \\ y_{i-1}^{1}= & c_{i}^{11} x_{0}^{1}+d_{i}^{11} y_{0}^{1}+f_{i}^{11} \\ x_{i}^{1}= & a_{i}^{11} x_{N}^{1}+e_{i}^{11} \\ y_{i}^{1}= & c_{i}^{11} x_{N}^{1}+d_{i}^{11} y_{N}^{1}+f_{i}^{11}\end{cases}
$$


$\forall i=K^{11}+1, \ldots, N$

$\forall i=1, \ldots, K^{21}$.

$$
\begin{cases}x_{i-1}^{1}= & a_{i-K^{11}}^{12} x_{0}^{2}+e_{i-K^{11}}^{12} \\ y_{i-1}^{1}= & c_{i-K^{11}}^{12} x_{0}^{2}+d_{i-K^{11}}^{12} y_{0}^{2}+f_{i-K^{11}}^{12} \\ x_{i}^{1}= & a_{i-K^{11}}^{12} x_{M}^{2}+e_{i-K^{11}}^{12} \\ y_{i}^{1}= & c_{i-K^{11}}^{12} x_{M}^{2}+d_{i-K^{11}}^{12} y_{M}^{2}+f_{i-K^{11}}^{12}\end{cases}
$$

$$
\begin{cases}x_{i-1}^{2}= & a_{i}^{21} x_{0}^{1}+e_{i}^{21} \\ y_{i-1}^{2}= & c_{i}^{21} x_{0}^{1}+d_{i}^{21} y_{0}^{1}+f_{i}^{21} \\ x_{i}^{2}= & a_{i}^{21} x_{N}^{1}+e_{i}^{21} \\ y_{i}^{2}= & c_{i}^{21} x_{N}^{1}+d_{i}^{21} y_{N}^{1}+f_{i}^{21}\end{cases}
$$

$\forall i=K^{21}+1, \ldots, M$

$$
\begin{cases}x_{i-1}^{2}= & a_{i-K^{21}}^{22} x_{0}^{2}+e_{i-K 21}^{22} \\ y_{i-1}^{2}= & c_{i-K^{21}}^{22} x_{0}^{2}+d_{i-K^{21}}^{22} y_{0}^{2}+f_{i-K^{21}}^{22} \\ x_{i}^{2}= & a_{i-K^{21}}^{22} x_{M}^{2}+e_{i-K^{21}}^{22} \\ y_{i}^{2}= & c_{i-K^{21}}^{22} x_{M}^{2}+d_{i-K^{21}}^{22} y_{M}^{2}+f_{i-K^{21}}^{22}\end{cases}
$$

where $d_{i}^{\alpha \beta}$ is a random variable.

In this way we obtain $a_{i}^{\alpha, \beta}, c_{i}^{\alpha, \beta}, e_{i}^{\alpha, \beta}, f_{i}^{\alpha, \beta}, \alpha, \beta \in\{1,2\}, i=1, \ldots, K^{\alpha \beta}$

$$
\begin{aligned}
& \left\{\begin{aligned}
a_{i}^{11} & =\frac{x_{i}^{1}-x_{i-1}^{1}}{x_{N}^{1}-x_{0}^{1}} \\
e_{i}^{11} & =\frac{x_{N}^{1} x_{i-1}^{1}-x_{0}^{1} x_{i}^{1}}{x_{N}^{1}-x_{0}^{1}} \\
c_{i}^{11} & =\frac{y_{i}^{1}-y_{i-1}^{1}}{x_{N}^{1}-x_{0}^{1}}-d_{i}^{11} \frac{y_{N}^{1}-y_{0}^{1}}{x_{N}^{1}-x_{0}^{1}} \\
f_{i}^{11} & =\frac{x_{N}^{1} y_{i-1}^{1}-x_{0}^{1} y_{i}^{1}}{x_{N}^{1}-x_{0}^{1}}-d_{i}^{11} \frac{x_{N}^{1} y_{0}^{1}-x_{0}^{1} y_{N}^{1}}{x_{N}^{1}-x_{0}^{1}}
\end{aligned}\right. \\
& \left\{\begin{aligned}
a_{i}^{12} & =\frac{x_{i}^{1}-x_{i-1}^{1}}{x_{M}^{2}-x_{0}^{2}} \\
e_{i}^{12} & =\frac{x_{M}^{2} x_{i-1}^{1}-x_{0}^{2} x_{i}^{1}}{x_{M}^{2}-x_{0}^{2}} \\
c_{i}^{12} & =\frac{y_{i}^{1}-y_{i-1}^{1}}{x_{M}^{2}-x_{0}^{2}}-d_{i}^{12} \frac{y_{M}^{2}-y_{0}^{2}}{x_{M}^{2}-x_{0}^{2}} \\
f_{i}^{12} & =\frac{x_{M}^{2} y_{i-1}^{1}-x_{0}^{2} y_{i}^{1}}{x_{M}^{2}-x_{0}^{2}}-d_{i}^{12} \frac{x_{M}^{2} y_{0}^{2}-x_{0}^{2} y_{M}^{2}}{x_{M}^{2}-x_{0}^{2}}
\end{aligned}\right. \\
& \left\{\begin{aligned}
a_{i}^{21} & =\frac{x_{i}^{2}-x_{i-1}^{2}}{x_{N}^{1}-x_{0}^{1}} \\
e_{i}^{21} & =\frac{x_{N}^{1} x_{i-1}^{2}-x_{0}^{1} x_{i}^{2}}{x_{N}^{1}-x_{0}^{1}} \\
c_{i}^{11} & =\frac{y_{i}^{2}-y_{i-1}^{2}}{x_{N}^{1}-x_{0}^{1}}-d_{i}^{21} \frac{y_{N}^{1}-y_{0}^{1}}{x_{N}^{1}-x_{0}^{1}} \\
f_{i}^{21} & =\frac{x_{N}^{1} y_{i-1}^{2}-x_{0}^{1} y_{i}^{2}}{x_{N}^{1}-x_{0}^{1}}-d_{i}^{21} \frac{x_{N}^{1} y_{0}^{1}-x_{0}^{1} y_{N}^{1}}{x_{N}^{1}-x_{0}^{1}}
\end{aligned}\right.
\end{aligned}
$$




$$
\left\{\begin{aligned}
a_{i}^{22} & =\frac{x_{i}^{2}-x_{i-1}^{2}}{x_{M}^{2}-x_{0}^{2}} \\
e_{i}^{22} & =\frac{x_{M}^{2} x_{i-1}^{2}-x_{0}^{2} x_{i}^{2}}{x_{M}^{2}-x_{0}^{2}} \\
c_{i}^{22} & =\frac{y_{i}^{2}-y_{i-1}^{2}}{x_{M}^{2}-x_{0}^{2}}-d_{i}^{22} \frac{y_{M}^{2}-y_{0}^{2}}{x_{M}^{2}-x_{0}^{2}} \\
f_{i}^{12} & =\frac{x_{M}^{2} y_{i-1}^{2}-x_{0}^{2} y_{i}^{2}}{x_{M}^{2}-x_{0}^{2}}-d_{i}^{22} \frac{x_{M}^{2} y_{0}^{2}-x_{0}^{2} y_{M}^{2}}{x_{M}^{2}-x_{0}^{2}}
\end{aligned}\right.
$$

Suppose ess sup $\max _{i} d_{i}^{\alpha \beta}<1$, for all $\alpha, \beta \in\{1,2\}$ and $i=1, \ldots, K^{\alpha, \beta}$.

Then $\Phi_{i}^{\alpha \beta}$ is a contraction and $\left\{\Omega \times \mathbb{R}^{2}, \Phi_{i}^{\alpha \beta}\right\}$ is a graph directed random iterated function system. We will prove that this graph directed random iterated function system satisfies the theorem.

Let

$$
\begin{aligned}
& C_{1}^{\omega}=\left\{f \mid f: \Omega \times\left[x_{0}^{1}, x_{N}^{1}\right] \rightarrow \mathbb{R}, f^{\omega}\left(x_{0}^{1}\right)=y_{0}^{1}, f^{\omega}\left(x_{N}^{1}\right)=y_{N}^{1}, \text { cont. a.s. }\right\} \\
& C_{2}^{\omega}=\left\{g \mid g: \omega \times\left[x_{0}^{2}, x_{M}^{2}\right] \rightarrow \mathbb{R}, g^{\omega}\left(x_{0}^{2}\right)=y_{0}^{2}, g^{\omega}\left(x_{M}^{2}\right)=y_{M}^{2}, \text { cont. a.s. }\right\}
\end{aligned}
$$

For $f_{1}, f_{2} \in C_{1}^{\omega}$ we define

$$
d_{\infty}^{*}\left(f_{1}, f_{2}\right)=e s s \sup _{\omega} d_{\infty}\left(f_{1}^{\omega}, f_{2}^{\omega}\right)
$$

where

$$
d_{\infty}\left(f_{1}, f_{2}\right)=\max _{x}\left\{\left|f_{1}^{\omega}(x)-f_{2}^{\omega}(x)\right|, x \in\left[x_{0}^{1}, x_{N}^{1}\right]\right\} .
$$

$\left(C_{1}^{\omega}, d_{\omega}^{*}\right)$ and $\left(C_{2}^{\omega}, d_{\omega}^{*}\right)$ are complete metric spaces, hence $C_{1}^{\omega} \times C_{2}^{\omega}$ is also a complete metric space with

$$
\begin{gathered}
\tilde{f}(\omega, x)=\left\{\begin{array}{lc}
C_{i}^{11} I_{i}^{-1}(x)+d_{i}^{11} f\left(\omega, I_{i}^{-1}(x)+f_{i}^{11}\right) & \text { if } x \in\left[x_{i-1}^{1}, x_{i}^{1}\right], \\
\left.C_{i-K^{11}}^{12} I_{i}^{-1}(x)+d_{i-K^{11}}^{12} g\left(\omega, I_{i}^{-1}(x)\right)+f_{i-K^{11}}^{12}\right) & i=1, \ldots, K^{11} \\
& \left.i=K^{11}+1, \ldots, N, x_{i-1}^{1}, x_{i}^{1}\right],
\end{array}\right. \\
\tilde{g}(\omega, y)=\left\{\begin{array}{lc}
C_{j}^{21} J_{j}^{-1}(y)+d_{j}^{21} f\left(\omega, J_{j}^{-1}(y)+f_{j}^{21}\right) & \text { if } y \in\left[x_{j-1}^{2}, x_{j}^{2}\right], \\
\left.C_{j-K^{21}}^{22} J_{j}^{-1}(y)+d_{j-K^{21}}^{22} g\left(\omega, J_{j}^{-1}(y)\right)+f_{j-K^{21}}^{22}\right) & j=1, \ldots, K^{21} \\
& \text { if } y \in\left[x_{i j-1}^{2}, x_{j}^{2}\right], \\
& j=K^{21}+1, \ldots, M,
\end{array}\right.
\end{gathered}
$$

where

$$
\begin{aligned}
& I_{i}:\left[x_{0}^{1}, x_{N}^{1}\right] \rightarrow\left[x_{i-1}^{1}, x_{i}^{1}\right], I_{i}(x)=a_{i}^{11} x+e_{i}^{11}, \text { for } i=1, \ldots, K^{11} \\
& I_{i}:\left[x_{0}^{2}, x_{M}^{2}\right] \rightarrow\left[x_{i-1}^{1}, x_{i}^{1}\right], I_{i}(x)=a_{i-K^{11}}^{12} x+e_{i-K^{11}}^{12}, \text { for } i=K^{11}+1, \ldots, N \\
& J_{i}:\left[x_{0}^{1}, x_{N}^{1}\right] \rightarrow\left[x_{i-1}^{1}, x_{i}^{1}\right], J_{i}(x)=a_{i}^{21} x+e_{i}^{21}, \text { for } i=1, \ldots, K^{21} \\
& J_{i}:\left[x_{0}^{2}, x_{M}^{2}\right] \rightarrow\left[x_{i-1}^{2}, x_{i}^{2}\right], J_{i}(x)=a_{i-K^{21}}^{22} x+e_{i-K^{21}}^{22}, \text { for } i=K^{21}+1, \ldots, M .
\end{aligned}
$$

We have

$$
\begin{aligned}
& \tilde{f}\left(\omega, x_{0}^{1}\right)=y_{0}^{1} \text { a. s., } \tilde{f}\left(\omega, x_{N}^{1}\right)=y_{N}^{1} \text { a. s. } \\
& \tilde{g}\left(\omega, x_{0}^{2}\right)=y_{0}^{2} \text { a. s., } \tilde{g}\left(\omega, x_{M}^{2}\right)=y_{M}^{2} \text { a. s. }
\end{aligned}
$$


One can show that $\tilde{f}$ and $\tilde{g}$ are continuous functions a.s.. We have to show that $T$ is a contraction.

$$
\begin{gathered}
d_{\infty}^{*}\left(f_{1}, f_{2}\right)=e s s \sup _{\omega} \max _{x}\left\{\left|f_{1}(\omega, x)-f_{2}(\omega, x)\right|\right\} \\
\max _{x \in\left[x_{0}^{1}, x_{K^{11}}^{1}\right]}\left\{\left|f_{1}(\omega, x)-f_{2}(\omega, x)\right|\right\}=\max _{i=1, \ldots, K^{11}}\left\{\left|d_{i}^{11}\right| \mid f_{1}\left(\omega, I_{i}^{-1}(x)\right)-\right. \\
\left.\left.-\quad f_{2}\left(\omega, I_{i}^{-1}(x)\right) \mid, x \in\left[x_{i-1}^{1}, x_{i}^{1}\right]\right\}\right\} \leq e s \sup _{\omega}\left\{d_{i}^{11}, i=1, \ldots, K^{11}\right\} \cdot d_{\infty}\left(f_{1}, f_{2}\right) \\
\max _{x \in\left[x_{K^{11}}^{1}, x_{M}^{1}\right]}\left\{\left|f_{1}(\omega, x)-f_{2}(\omega, x)\right|\right\}=\max _{i=K^{11}+1, \ldots, N}\left\{\left|d_{i-K^{11}}^{12}\right| \mid g_{1}\left(\omega, I_{i}^{-1}(x)\right)-\right. \\
\left.\left.g_{2}\left(\omega, I_{i}^{-1}(x)\right) \mid, x \in\left[x_{i-1}^{1}, x_{i}^{1}\right]\right\}\right\} \leq e s s \sup _{\omega}\left\{d_{i}^{12}, i=1, \ldots, K^{12}\right\} \cdot d_{\infty}\left(f_{1}, f_{2}\right) \\
d_{\infty}^{*}\left(f_{1}, f_{2}\right) \leq \max \left\{e s s \sup _{\omega}\left\{d_{i}^{12}, i=1, \ldots, K^{12}\right\}, e s s \sup _{\omega}\left\{d_{i}^{11}, i=1, \ldots, K^{11}\right\}\right\} . \\
\max \left\{d_{\infty}^{*}\left(f_{1}, f_{2}\right), d_{\infty}^{*}\left(g_{1}, g_{2}\right)\right\}
\end{gathered}
$$

similarly

$$
\begin{aligned}
d_{\infty}^{*}\left(g_{1}, g_{2}\right) \leq & \max \left\{\text { ess } \sup _{\omega}\left\{d_{i}^{21}, i=1, \ldots, K^{21}\right\}, \text { ess } \sup _{\omega}\left\{d_{i}^{22}, i=1, \ldots, K^{22}\right\}\right\} . \\
& \cdot \max \left\{d_{\infty}^{*}\left(f_{1}, f_{2}\right), d_{\infty}^{*}\left(g_{1}, g_{2}\right)\right\} .
\end{aligned}
$$

So

$$
\begin{aligned}
d\left(T\left(f_{1}, g_{1}\right), T\left(f_{2}, g_{2}\right)\right) & =\max \left\{d_{\infty}^{*}\left(\tilde{f}_{1}, \tilde{f}_{2}\right), d_{\infty}^{*}\left(\tilde{g}_{1}, \tilde{g}_{2}\right)\right\} \leq \\
& \leq r \cdot \max \left\{d_{\infty}^{*}\left(f_{1} \cdot f_{2}\right), d_{\infty}^{*}\left(g_{1}, g_{2}\right)\right\}
\end{aligned}
$$

where

$$
\begin{aligned}
r= & \max \left\{\operatorname{ess}_{\omega}\left\{d_{i}^{21}, i=1, \ldots, K^{21}\right\}, \text { ess } \sup _{\omega}\left\{d_{i}^{22}, i=1, \ldots, K^{22}\right\}\right. \\
& \left.\quad \text { ess } \sup _{\omega}\left\{d_{i}^{12}, i=1, \ldots, K^{12}\right\}, \text { ess } \sup _{\omega}\left\{d_{i}^{11}, i=1, \ldots, K^{11}\right\}\right\}<1 .
\end{aligned}
$$

Using Banach fixed point theorem, $T$ has a unique fixed point $\left(f_{0}, g_{0}\right)$ :

$$
T\left(f_{0}, g_{0}\right)=\left(f_{0}, g_{0}\right) \text {. }
$$

Let $F$ and $G$ be the graph of $f_{0}$ and $g_{0}$ :

$$
\begin{aligned}
& f_{0}\left(\omega, a_{i}^{11} x+e_{i}^{11}\right)=c_{i}^{11} x+d_{i}^{11} f_{0}(\omega, x)+f_{i}^{11} \text { for } i=1, \ldots, K^{11} \\
& f_{0}\left(\omega, a_{i}^{12} y+e_{i}^{12}\right)=c_{i}^{12} y+d_{i}^{12} g_{0}(\omega, y)+f_{i}^{12} \text { for } i=1, \ldots, K^{12},
\end{aligned}
$$

which imply:

$$
F=\bigcup_{i=1}^{K^{11}} \Phi_{i}^{11}(F) \cup \bigcup_{i=1}^{K^{12}} \Phi_{i}^{12}(G)
$$

similarly

$$
G=\bigcup_{i=1}^{K^{21}} \Phi_{i}^{21}(F) \cup \bigcup_{i=1}^{K^{22}} \Phi_{i}^{22}(G)
$$


According to the uniqueness of the solution, the graph of $f_{0}$ and $g_{0}$ are the attractor of the fractal interpolation function.

In the last few years the method of fractal interpolation was widely used in signal processing, computer geometry, image compression and of course in approximation theory. The stochastic type fractal interpolation method and the graph-directed random fractal interpolation function present more flexibility and therefore it can be applied much better in the case of real data interpolation.

\section{References}

[1] Barnsley, M.F., Fractal functions and interpolation, Constructive Approximation, 2(1986), 303-329.

[2] Barnsley, M.F., Fractals Everywhere, Academic Press, 1993.

[3] Barnsley, M.F., Demko, S., Iterated function systems and the global construction of fractals, Pro. Roy. Soc. London, A399(1985), 243-275.

[4] Chand, A.K.B., Kapoor, G.P., Generalized cubic spline interpolation function, SIAM J. Numer. Anal., 44(2006), no. 2, 655-676.

[5] Deniz, A., Özdemir, Y., Grapd-directed fractal interpolation functions, Turk. J. Math., 41(2017), 829-840.

[6] Edgar, G., Measure, Topology and Fractal Geometry, Springer, New York, 2008.

[7] Hutchinson, J.E., Rüschendorf, L., Selfsimilar fractals and selfsimilar random fractals, Progress in Probability, 46(2000), 109-123.

[8] Nevascués, M.A., Sebastián, M.V., Generalization of Hermite functions by fractal interpolation, J. Approx. Theory, 131(2004), no. 1, 19-29.

[9] Wang, H.Y., Yu, J.S., Fractal interpolation functions with variable parameters and their analytical properties, J. Approx. Theory, 175(2013), 1-18.

Ildikó Somogyi

Babeş-Bolyai University,

Faculty of Mathematics and Computer Sciences,

1, Kogălniceanu Street,

400084 Cluj-Napoca, Romania

e-mail: ilkovacs@math. ubbcluj .ro

Anna Soós

Babeş-Bolyai University,

Faculty of Mathematics and Computer Sciences,

1, Kogălniceanu Street,

400084 Cluj-Napoca, Romania

e-mail: asoos@math.ubbcluj.ro 